\title{
A Self-adaptive Reconfiguration Scheme for Throughput Maximization in Municipal WMNs
}

\author{
Bing Leng ${ }^{\mathrm{a}, \mathrm{b}, *}$, Liusheng Huang ${ }^{\mathrm{a}, \mathrm{b}}$, Hongli Xu ${ }^{\mathrm{a}, \mathrm{b}}$, Chenkai Yang ${ }^{\mathrm{a}, \mathrm{b}}$, Xinglong Wang ${ }^{\mathrm{a}, \mathrm{b}}$ \\ ${ }^{a} 96$ Jinzhai Road, School of CS \& Tech., USTC, Hefei, 230027, China \\ ${ }^{b} 188$ Renai Road, Suzhou Institute for Advanced Study, USTC, Suzhou, 215123, China
}

\begin{abstract}
Multi-hop wireless mesh network (WMN) is considered to be an economical technique for last-mile broadband Internet access [1]. Fueled by the rapid development of the Internet of Things, it is increasing important to provide robust and high-quality urban wireless mesh networks(WMNs) for public service. However, WMNs encounter frequent link failures during their lifetime in practical use as a consequence of large-scale wireless interference, block buildings, etc., which deeply influences the performance of the network. These failures should be recovered in time or prevented with foresight. Tremendous labor and management cost should also be avoided. In this paper, we focus on an interesting phenomenon called "loop effect" in urban WMNs which may cause frequent link failures. A scheme named anti-loop network reconfiguration system(ALRS) for link recovery in municipal WMNs with QoS requirement is proposed and analyzed. ALRS takes the historical information into consideration to achieve maximum throughput, interference avoidance and self-reconfiguration with no requirement on mesh node antennas. Our evaluation results show that ALRS outperforms all the existing techniques in improving network throughput and reducing the occurrence probability of continuous link failure.
\end{abstract}

Keywords: reconfiguration; throughput; municipal WMN; optimal

* Corresponding author

Email address: lengb@mail . ustc . edu . cn (Bing Leng)

The abstract of this paper [2] is shown in IEEE/ACM International Symposium on Quality of Service (IWQoS) 2015 as a poster.

Preprint submitted to Journal of $\mathrm{BT}_{E} X$ Templates

December 23, 2015

(C) 2016. This manuscript version is made available under the Elsevier user license http://www.elsevier.com/open-access/userlicense/1.0/ 


\section{INTRODUCTION}

Wireless mesh networks (WMNs) are being used and deployed widely all around the world for various reasons such as public safety, environmental monitoring and citywide wireless Internet services [3, 4]. Although faced with some difficulties, WMN is thought to be a preferred municipal Internet service provider [5] with huge potential due to its automatic connection, ease of installation, dynamic route discovery, flexibility and other advantages. Meanwhile, people benefit from the transformations of WMNs(e.g., using multi-radio/multi-channel systems [6, 7, 8]) in utilizing it with different purposes, among which, municipal network with higher quality of service (QoS) is the most common application scenario, and municipal WMNs experience increasing interest and attention in providing quality-guaranteed cost-efficient services. For example, Singapore and the city of Cambridge intend to construct city-wide ubiquitous Internet access with WMNs[9], and China is also planning to provide municipal WMN$\mathrm{s}$ in plenty of cities. The research on providing QoS-guaranteed municipal WMNs is in urgent need.

Because of the natural defects of the wireless transmission, deploying municipal WMNs is faced with challenges such as guaranteeing robustness and avoiding interference. Meanwhile, constraints like high throughput, robustness, interference avoidance and financial restriction must be considered simultaneously as well when we are trying to provide city-wide Internet access. In this case, a multi-channel multi-radio scheme (mr-WMN) mentioned in several studies [1, 9, 10, 11, 12] is considered to be a suitable choice to meet all the requirements above-mentioned. Instead of a single antenna, the mesh node (or router, AP) is equipped with two or even more antennas to achieve simultaneous message exchanging among several adjacent mesh nodes by selecting orthogonal channels appropriately. mr-WMN scheme is good at achieving high throughput with flexibility and expandability of the network maintained. But on the other hand, it is also criticized for its high link failure frequency. This is due to the natural risk of link failure and interference in wireless transmission and mr-WMN indeed aggravates it. There are some works[11, 12] concentrating on making a quick and automatic recovery from link failures caused by "ripple effect" in mr-WMNs, but 
their works do not pay attention to another serious problem that is mainly occurred in urban area - the "loop effect".

The "loop effect" is also a phenomenon in describing the occurrence of frequent link failures - but occurs in urban areas and is mainly caused by the regularity of city 35 activities. In the city, people are more likely to be gathered due to some periodical reasons, such as at the main streets during the rush hour, at a museum at the weekend, etc. These phenomena generate a huge burden as well as interference area to wireless transmission because people are mostly carrying their cellphones. On the other hand, unexpected blocks such as newly constructed buildings may influence the wireless transmission as well, which may lead to unpredictable or long-time link failures. These influences are human related and common in urban area. We propose and define the "loop effect" as the frequent link failures caused by human activities in the urban area. According to our knowledge, it is the first time to consider the influence of human characters to the performance of WMNs.

Numerous works have been proposed to deal with the interference issue in $\mathrm{mr}-$ WMNs, among which, recovery from link failures [13, 14] is most widely studied with plenty of solutions presented. There are three important rules to follow both in link recovery and pursuing robustness:

1 Persistence Requirement: The network should not stop serving after deployed. If recovery needs manual operation, the interruption of network service is inevitable

2 Financial Constraint: Without self-reconfiguration mechanism, non-ignorable time and labor cost are unavoidable during the recovery of the network and cost extra expenses.

3 Fluency Requirement: The network may not experience frequent indisposed service, due to which the frequency of link failures should be limited.

The latest works [11, 12] on link recovery in mr-WMNs have already met the first two conditions. They succeed in maintaining the persistence of network services and keeping link recovery manual-free. However, this is not enough to bring the municipal 
WMNs to our daily life. We found that they experienced more link failures than expected during the simulation, and the reason is a common phenomenon in our daily life - that interference sometimes causes vast overlay for its periodic occurrence in urban areas - namely the "loop effect". Unfortunately, previous works paid no attention on human activities and in turn suffered from the "loop effect" badly.

In order to eliminate the "loop effect" and keep the former two conditions maintained simultaneously, we present a mechanism for real-time link recovery with robustness guaranteed and maximum throughput achieved in municipal WMNs named Anti-Loop Network Reconfiguration System (ALRS). The advantages of our work can be summarized as follows:

- We combine urban characteristics with the interference avoidance in the consideration of link recovery.

- ALRS estimates local urban characteristics as soon as the link failure is discovered, and reduces the occurrence possibility of "loop effect".

- The network recovery is cost-effective because of the local limitation of topology changes in ALRS.

- ALRS achieves throughput maximization after the recovery.

The rest of this paper is organized as follows. Section II and III explain relevant concepts and related works. We present the cause and characteristic of "loop effect" in Section IV. The detailed design of ALRS and evaluation results are given in Section V and VI. Section VII concludes the paper.

\section{MODELs and CONCEPTs}

In this section, we first present a brief introduction to the multi-channel multi-radio wireless mesh network system (mr-WMN). Then, several definitions involved in ALRS are explained respectively. 


\subsection{Multi-channel Multi-radio WMN}

Multi-channel multi-radio wireless mesh networks, known as mr-WMNs in some papers [11, 12], are thought to be the preferred choice for providing everywhere Internet access with QoS supports. There are two components in mr-WMNs - the mesh nodes and the gateway - in terms of hardware. The router (or AP) plays the role of a mesh node in mr-WMNs, which is responsible for transferring messages to other mesh nodes or the gateway as well as providing Internet access in its signal cover. Contrary to wireless transferring, a gateway is connected to the Internet with a wire-line infinite-bandwidth link. A gateway is a mesh node with an extra, interference-ignored, unlimited access to the Internet, in a sense. All the Internet services are essentially provided by the gateway through multi-hop mesh node paths. An example topology of mr-WMN can be seen in Fig 1

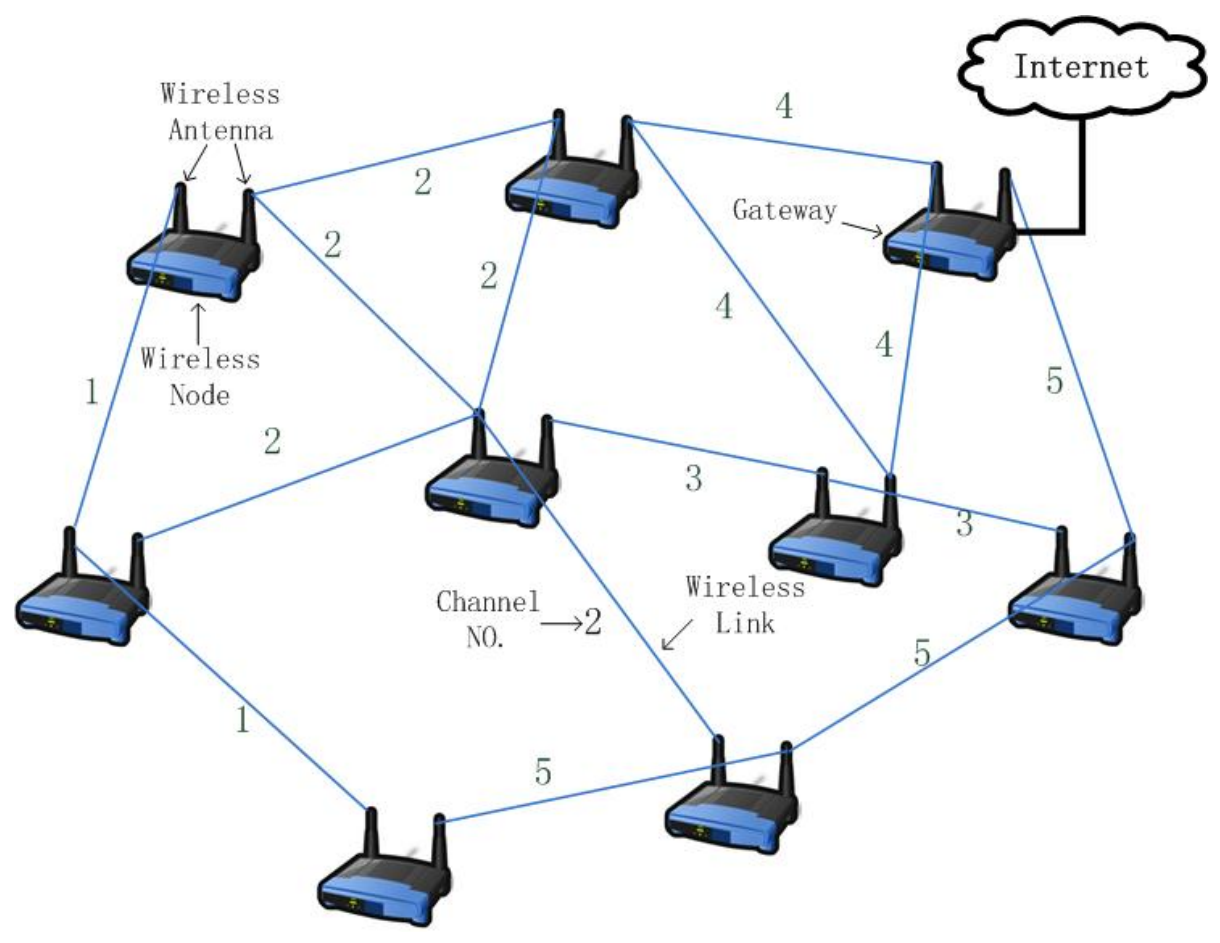

Figure 1: An Example Topology of mr-WMN

The number of antennas (or radios, radio interface cards) is not restricted on a 
mesh node, indicating it is able to maintain several transmissions synchronously. Mesh nodes can communicate with each other if and only if at least one pair of their antennas is working in the same channel.

There are $\mathscr{K}$ orthogonal channels available for the network, as $\mathscr{K}$ can be any positive integer for universality. Actually, the transmission volume of a gateway and the number of antennas on a node are both limited, and there are other serious problems in deploying and utilizing large-scale WMNs(e.g. the interference generated within the network is extremely aggravated and should not be ignored, the giant demand of transmission power, etc.), which will not be discussed in this paper. In this case, although the assumption is unrestricted, our analysis and mechanism are presented under the precondition that the network scale is limited. This is reasonable because the urban WMNs are deployed in terms of blocks and streets at most.

\subsection{Definition of Several Concepts}

\subsection{1. link}

A gateway is regarded as a mesh node when it is exchanging information with mesh nodes.[11, 12]

If at least one pair of antenna within communication range from two nodes is working in exactly one channel, there is a link between two nodes. Link is the only manner of transmission no matter in node-node or node-gateway transmission.[13]

\subsection{2. link failure}

When two nodes fail in transmitting messages as they used to, the link between these nodes is considered to be failed. The cause of a link failure is various and the mostly common reasons for the link failure are mainly interference \& link requirement violation.[11, 12]

\subsection{3. throughput}

The throughput of the network in this paper is the sum of bandwidth that mesh nodes can provide to users [5]. The higher the throughput is, the more information users are able to obtain from the Internet. 


\subsection{4. transmission \& interference range}

When a wireless signal transmission device $\mathscr{A}$ is functioning, all the wireless devices in its transmission range [15] are able to recognize the messages that $\mathscr{A}$ sends. In this case, these devices have the ability to communicate with $\mathscr{A}$. But A also has an interference range [16], which is generally more than twice as the transmission range and all the devices are influenced within it. In other words, these devices are interfered by $\mathscr{A}$, if they are using the very channel that $\mathscr{A}$ is working on. Interference is bidirectional, and all the participants in interference are influenced equally, which is not acceptable in wireless communication.

\subsection{5. priority}

In our mechanism, the gateway is responsible for the network information storage, link quality collection and reconfiguration plan generation. Each link in the network has a list of channels stored at the gateway, indicating its channel priority. The priority is the faithful reflection of channel performance. The smaller the priority is, the better performance the link can achieve on the corresponding channel.

\section{RELATED WORK}

Wireless mesh network catches plenty of attentions from researchers as a common tool for providing efficient wireless Internet access for citizens. More and more scholars have focused their sights on performance of municipal WMNs. The authors in [9] present a scheme for router deployment in WMN to achieve budget control, connectivity and system robustness simultaneously. A paper [1] focusing on throughput maximization and fair bandwidth allocation in WMNs is also widely cited by other works. There are vast quantities of researches concentrating on quality of service (QoS).

Link recovery is an important research topic in WMN to meet QoS requirements. The performance of wireless mesh network drops down significantly while encountering link failures. Among various solutions proposed for recovery from link failures in WMNs, four categories can be summarized according to their basic ideas. These techniques for link recovery are listed as follows. 


\subsection{Initial Resource Allocation}

155 belong to this category. They mostly offer a network topology or a routing method or a channel assignment to satisfy diverse QoS guarantees.

Among these achievements, Alicherry, Bhatia and Li [5] proposed a representative joint channel assignment solution. Their method takes interference and channel availability into account to formulate channel assignment and routing problem mathematically and efficiently. The advantage of their technique is the maximization of throughput and effective exploitation of the increased number of channels and radios.

A paper based on the natural multi-radio potential of WMNs written by Brzezinski, Zussman and Modiano [17] makes use of the local pooling notion in distributed mechanism accomplishing $100 \%$ throughput of the network.

The Initial Resource Allocation algorithms perform optimal and comprehensive on utilizing the advantages of wireless mesh networks. But as a cost of outstanding performance, they mostly require global configuration of the network changes, which makes them inappropriate and inefficient for recovery from link failures.

\subsection{Interference Aware}

Interference aware[18] is also a widely used method for link recovery. Ramachandran et al. [7 put forward a method for solving the problem of interference in multiradio wireless mesh network. They proposed a novel mechanism for interference definition and a channel assignment protocol based on it. Their system pointed out an insightful interference estimation scheme that could be deployed in all wireless mesh networks.

Inspired by their solution, we modified their idea of interference estimation by taking historical information into consideration for further utilization.

\subsection{Greedy Algorithm}

Unlike Initial Resource Allocation, greedy algorithms in link recovery focus their attention basically on local benefit instead of the global effectiveness. Obviously, greedy methods have lower algorithm complexity than other algorithms by concerning 
the vicinity of the faulty link(s) simply. [19] presents a distributed algorithm making dynamic channel assignment according to the traffic loads. It can handle both network link failure as well as traffic load changes simultaneously.

Though so many advantages they have, greedy algorithms suffer from the "ripple effect" badly. While recovering from one link failure, greedy algorithms may cause other links functioning regularly fail sequentially, which seriously affects the performance of the whole network.

\section{4. $A R S \& E R S$}

Kim and Shin [11] give a new method namely Autonomous network Reconfiguration System (ARS) for solving link recovery problem in WMNs. ARS collects information and lists all feasible plans for network reconfiguration when a link failure is detected. Instead of global changing, ARS keeps the changes within a range around the faulty link. Meanwhile, ARS distinguishes the available plans with QoS test to avoid the ripple effect. An optimal plan will be picked by an optimal plan selection method at last. The process can be seen in Fig 2 .

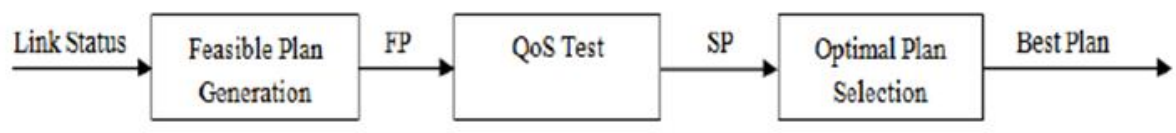

Figure 2: ARS Operation Process

Enhanced Reconfiguration System (ERS) [12] is an improvement of ARS by taking the cost of changes into consideration. With the definition of cost of each change in network topology, ERS wisely chooses the optimal plan to make the network recover from link failure at the minimum cost.

Although ARS and ERS have been widely used for link failure recovery, they may suffer terrible experience for characteristic inadequacy in the urban area. While they are trying to make full use of all the resources, the optimal plan selection schemes of ARS and ERS fail in reflecting human activities which guides to a non-optimal (or even worse) plan. 


\section{LOOP EFFECT}

Just like the "ripple effect"[20] is discovered during the practical use, the performance of practical municipal WMN is influenced by rhythmic interferences or requireof links are detected and collected by a distributed algorithm running on every node and reported to the gateway periodically. The gateway is responsible for storing all the link information respectively and generating corresponding localized reconfiguration plans. 
The optimal plan is selected by a scheme deployed on the gateway as well, which will

with the available throughput-maximum channels, which can be achieved by ALRS' exhaustive design. Running an initial-resource-allocation algorithm is the final action of the initial phase to assign the rest links [10, 17]. The detailed process of ALRS is about to be introduced following.

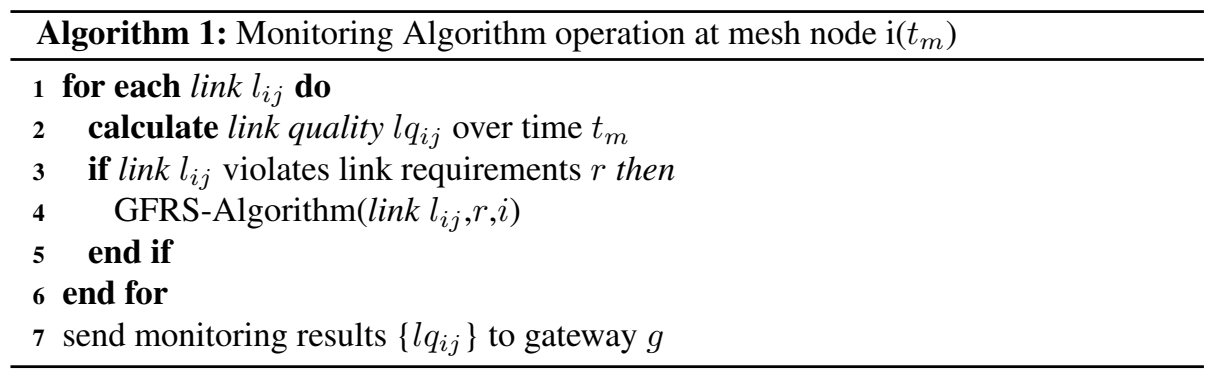

Algorithm 12/3 depict the operation of mesh node.

Firstly, mesh nodes are designed to monitor link states at every timeslot $t_{m}$ (e.g., 10s) when the network is functioning normally. All the link states collected should be reported to the gateway at the end of $t_{m}$ cyclically. Secondly, when a link violates 


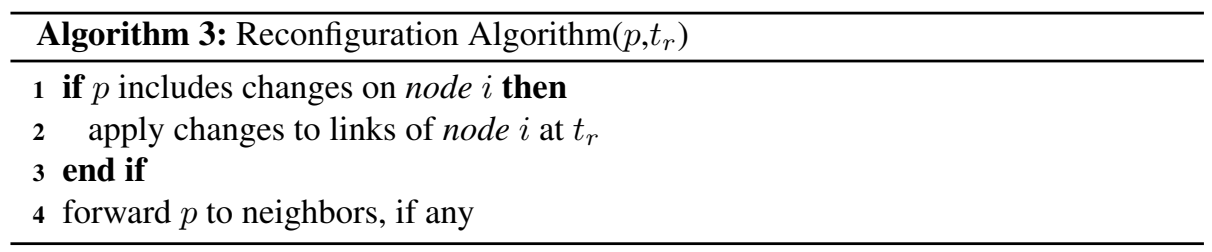

link requirement, the discoverer of the link failure calls for a leader election among local mesh routers that use a faulty channel. The leader node is elected by the bully algorithm [21] and is responsible for sending the reconfiguration request to the gateway. At this time, the gateway generates a corresponding reconfiguration plan $p$ according to its knowledge as a response. Finally, as soon as a mesh node (including the leader node) receives $p$ and has not applied it yet, it applies the changes and forwards $p$ to its neighbors.

Actually, we could run the leader election algorithm in Algorithm 2 in advance to make the local leader also the command node as the gateway does, which makes our mechanism completely distributed. But firstly, this requires storing memory and relatively strong calculating capability, which is usually not available to a normal mesh node. Secondly, both states transmission and storage lead to further cost since the data could be collected incidentally with the normal data towards the gateway, which only requires small additional bandwidth cost. Thirdly, the gateway is much more unlikely to go wrong than a mesh node. Hence the gateway is preferred to be the commander of ALRS.

\subsection{Priority List Update Method}

The link states gather at the gateway, where the link qualities and link failure record are stored separately. The link qualities are used as constraints for feasible reconfiguration plan selection to prevent "ripple effect", which is detailed discussed in [11].

On the other hand, if link $l$ working on channel $c$ fails, the gateway triggers faultycount module to update the priority list, whose storage structure is shown in Fig 3

We can assume priority of channel $c$ of link $l$, latest failure time, continuous failure count, current time as $p_{l, c}, t_{l, c}, c_{l, c}$ and $t_{c u r r e n t}$ respectively. These values are stored at the gateway. When a link $l$ on channel $c h$ is detected failed, the priority list update 


\begin{tabular}{|l|l|l|l|l|}
\hline Link ID & Channel No. & Priority & Latest Failure Time & Continious Failure Count \\
\hline
\end{tabular}

Figure 3: Priority Storage Information

method is awoken. Firstly, the gateway checks the current time $t_{\text {current }}$ and the latest

failure detected time $t_{l, c}$ of link $l$. If the time interval between these two parameters is less than a preset threshold $T$, ALRS identifies it as a continuous failure. In this case, for all the available channels on link $l$, their counters $c_{l, c} s$ as well as the global continuous failure counter count should be increased by 1 . Then ALRS updates the priority list of link $l$ as $p_{l, c}=p_{l, c} * \alpha * c_{l, c}$, the latest failure detected time list of link $l$ as $t_{l, c}=t_{\text {current }}$. In the formula, $\alpha$ is used for representing the proportion of the original priority, indicating the sensitiveness of the mechanism to link failures. Meanwhile, the local environmental influence is also considered in ALRS. Since the link failure is detected on channel $c h$, all the priority lists of the links within the interference area of link $l$ are updated as well. At this time, not all the channels but only the detected failure channel $c h$ is concerned, namely $p_{l^{\prime}, c}=p_{l^{\prime}, c} * \alpha^{*} c_{l^{\prime}, c h}$. At last, if continuous link failure counter count is discovered larger than a preset threshold count $_{\text {max }}$, ALRS identifies the link $l$ is not suitable for data transmission any more and calls for a route switch. The above process is shown in Algorithm 4

According to the Algorithm 4, the priority of different channels used by relevant links are considered comprehensively instead of unilaterally, which is a significant improvement achieved by ALRS. The priority $p_{l, c}$ is influenced by not only its own link state but also its neighbors operating conditions. Due to the benefit of our design, ALRS is more sensitive to local environmental changes than all the existing methods. The priority number rises when link failure happens, suggesting the degree of priority is decreased. Typically, it increases in factorial level if encountering continuous link failures no matter on either channel. When the network encounters a link failure, the $p_{l, c} \mathrm{~s}$ of all the links within the scope of interference distance from the failure link are updated.

The priority list is the result of sorting by priorities, according to which ALRS selects the best reconfiguration plan. 


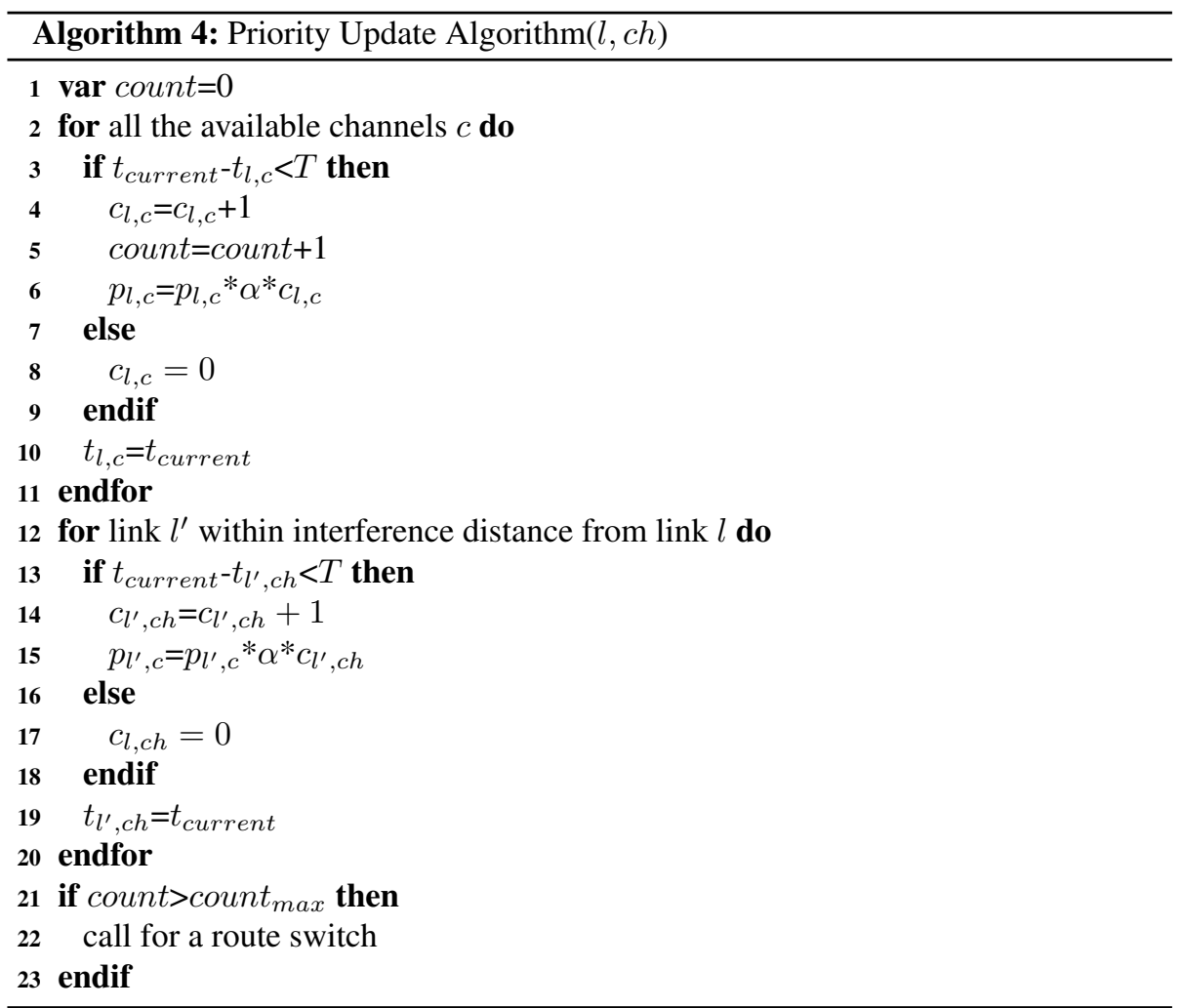

\subsection{Reconfiguration Plan Generation System \& Optimal Plan Selection}

There are three elementary operations that are used to make network topology reconfiguration with QoS guaranteed, namely the channel switch, radio switch and route switch [11, 12].

As shown in Table 11 three kinds of operations are exhibited individually. They are the components of the reconfiguration plan. It is a prerequisite that the discovered faulty channel should not be used again. At this time, a channel switch is necessary. Meanwhile, the reconfiguration may need a radio switch since the network topology may be quite different from the original one, and the reconfiguration plan should call for radio switches to maintain the network transmission during the reconfiguration. At the same time, if one link is discovered not suitable for transmission(as shown in Algorithm 47, a route switch is necessary. With these operations, each reconfiguration plan is able to be executed automatically with QoS guaranteed. 
Table 1: Formalization of Link Operations

\begin{tabular}{|c|c|}
\hline $\begin{array}{c}\text { Primitive } \\
\text { Changes }\end{array}$ & Description \\
\hline $\begin{array}{c}\text { Channel Switch } \\
\left(S\left(\mathscr{A}_{i}, \mathscr{B}_{j}\right)_{\alpha \rightarrow \beta}\right)\end{array}$ & $\begin{array}{c}\text { Radios } \mathscr{A}_{i} \text { and } \mathscr{B}_{j} \text { of link } \mathscr{A} \mathscr{B} \text { switch } \\
\text { their channel }(\alpha) \text { to channel }(\beta)\end{array}$ \\
\hline $\begin{array}{c}\text { Radio Switch } \\
\left(R\left(\mathscr{A}_{i}, \mathscr{B}_{j}\right)_{\alpha \rightarrow \beta}\right)\end{array}$ & $\begin{array}{c}\text { Radio } \mathscr{A}_{i} \text { in node } \mathscr{A} \text { re-associate with } \\
\text { radio } \mathscr{B}_{j} \text { in node } \mathscr{B} \text { using channel }(\beta)\end{array}$ \\
\hline $\begin{array}{c}\text { Route Switch } \\
\left(D\left(\mathscr{A}_{i}, \mathscr{B}_{j}\right)\right)\end{array}$ & $\begin{array}{c}\text { Radios } \mathscr{A}_{i} \text { and } \mathscr{A}_{j} \text { terminate link } \mathscr{A} \mathscr{B} \\
\text { and look for a detour path for } \\
\text { association }\end{array}$ \\
\hline
\end{tabular}

However, the network changes should be limited as locally as possible. Therefore,

2 and collected during the leader election process. The feasible plan generation is illustrated in Fig 4.

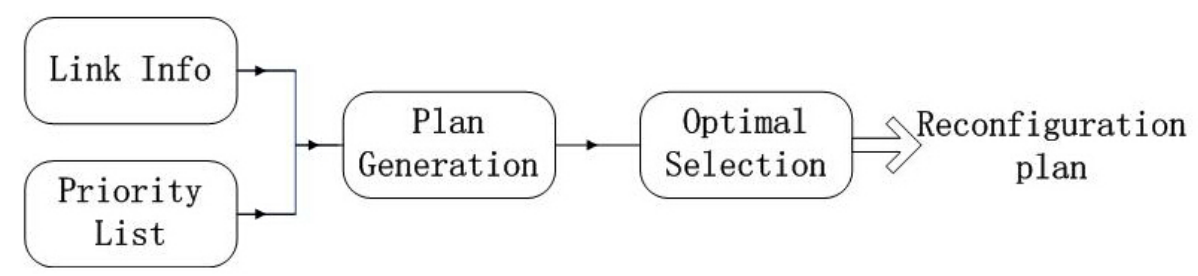

Figure 4: ALRS Process

ALRS now has at least one feasible reconfiguration plan which is QoS satisfiable. The gateway has to select an optimal plan if there are more than one. Our goals are achieving network maximum throughput as well as the min-max priority of all nongateway links. If any link connected to the gateway is involved in the reconfiguration plan except for calling for a route switch, the maximal available bandwidth channels should be allotted to it. Then the min-max priority can be obtained by selecting the min- 
max priority plan according to the priority list introduced in sub-section 5.3 . When

Definition 3. The set of links is $L=\left\{l_{i, j} \mid i, j \in \mathbb{Z}^{+}, i \neq j\right\}$.

Considering the bandwidth occupation:

Definition 4. The maximum bandwidth available on node $n_{i}$ is $b_{n_{i}}$

Our objective is to prove $\Sigma_{n_{i} \in N} b_{n_{i}}$ is maximum in our mechanism. 
Theorem 1. The total throughput achieves maximum in ALRS.

Proof. For a random node $n_{i} \in N$, it has inside links $l_{i, j_{1}}, l_{i, j_{2}}, \ldots, l_{i, j_{m}}$ and outside links $l_{k_{1}, i}, l_{k_{2}, i}, \ldots, l_{k_{n}, i}$, where $m$ and $n$ stand for the number of its inside and outside links. Considering a given time point, the real-time bandwidth occupation of all the inside and outside links of node $n_{i}$ could be represented as $O_{i, j_{1}}, O_{i, j_{2}}, \ldots, O_{i, j_{m}}$ and $O_{k_{1}, i}, O_{k_{2}, i}, \ldots, O_{k_{n}, i}$, and at this very time point, the maximum bandwidth node $i$ could provide is

$$
b_{n_{i}}=\Sigma_{n_{j} \in N, i \neq j} O_{i, j}-\Sigma_{n_{k} \in N, i \neq k} O_{k, i} \quad\left(n_{i} \in N\right)
$$

This formula is also correct for other nodes. And if we formulate them and add them together, we could get

$$
\Sigma_{n_{i} \in N} b_{n_{i}}=\Sigma_{s_{j} \in S, n_{k} \in N} O_{k, j}
$$

Namely the total bandwidth provided in WMN equals to the sum of occupied bandwidth of the links connected to the gateway. This is because except for the inside links of the gateways, other links are both inside links of one node and outside links of another one, making them eliminated in the calculation. In this case, we can get access to the following lemma:

Lemma 1. For uplink transmission, the maximum throughput of a WMN equals to the sum of the capacity of the links connected to gateways.

It is similar to achieve the same lemma for downlink transmission. In this case, we could come to a conclusion that the maximum throughput of a WMN equals to the sum of the capacity of the links connected to gateways.

In the initial algorithm (seen in sub-section 5.2), the combined capacity of all gateway links achieves maximum, indicating the total bandwidth reaches maximum after initialization.

The topology of the network will not change except for reconfiguration. If the links connected to the gateway are not involved in the reconfiguration plan, the bandwidth maintains optimal. Otherwise, available largest bandwidth channels will be allotted to 
the links connected the gateway according to sub-section 5.4. and the network achieves its available maximum bandwidth either.

To sum up, the total throughput of the network achieves maximum in ALRS during its lifetime.

\subsection{Additional Comments}

The complexity of ALRS is reasonable thanks to the distributed and localized design. First, the monitoring module consumes less than $12 \mathrm{~Kb} / \mathrm{s}$ probing bandwidth for each radio. In addition, group formation incurs only $O(n)$ message overhead, where $n$ represents the size of the group. Moreover, the reconfiguration plan generation receives acceptable overhead as the changes are limited locally. Specifically, the optimal plan selection only incurs $O(l)$ complexity, in which $l$ indicates the number of links within $\mathscr{K}$ range from the failed link. Also, the priority list is the only information that needs to be stored at the gateway permanently, which takes $O(l)$ complexity.

The design of ALRS is not suitable for large scale WMN(neither is ARS and ERS). In urban WMNs, a gateway is mostly serving a block or even only part of it. This is because it may lead to congestion at the gateway if too many mesh nodes are supported by it. The network also faces higher risk in providing service since a gateway goes wrong will cause a larger scale of service unavailable. In this case, ALRS is practical, and both the collection and storage overhead are acceptable.

\section{PERFORMANCE EVALUATION}

In this section, we exhibit the performance of ALRS via simulation in large-scale network settings under various conditions to confirm that ALRS is able to detect link failures effectively in the network and achieve self-adaptive reconfiguration. We also compare the real-time throughput of ALRS and other techniques.

The introduction of our simulation environment is given in this section at first. Then we describe the reconfiguration cost and the detailed analysis of the simulation results comes last. 


\subsection{Simulation Model \& Method}

400 grid topology is used in which there are 25 nodes in the square of $1 * 1 \mathrm{~km}^{2}$. The distance between two adjacent nodes in our topology is $185 \mathrm{~m}$. The nodes are equipped with several radios, mainly based on their proximity to the gateway, and the number of radios on the nodes ranges from 1 to 4 randomly. We set the reconfiguration range in each interference node is not limited in our simulation. If an interference node is broadcasting in all the available channels it means there is a block at the very location of the node. The topology of block simulation is shown in Fig 5 , in which red links are the influenced links.

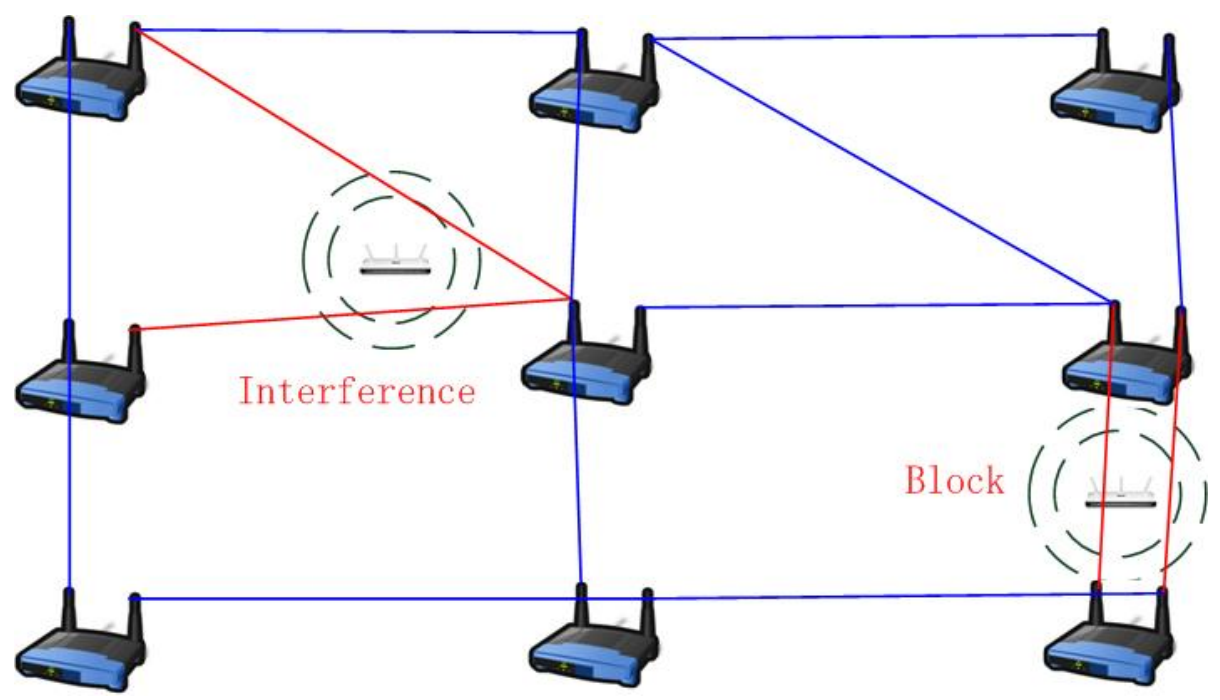

Figure 5: Block \& Interference Simulation $\mathscr{H}=3$ as the initial reconfiguration range.

There are several extra nodes in the topology as the interference sources, whose information is not available to the gateway. The interference nodes are randomly placed in the topology and their locations are changing from time to time. Meanwhile, interference nodes are broadcasting messages using either part or all of the available channels indicated by 802.11 protocols during the simulation. The number of radios

In the simulation, each node is allotted with a random number around a fixed val- 
ue as the traffic load, indicating the UDP flow gathered at the node. Routing policy is determined by the routing protocol multiradio-aware routing metric (WCETT [23]) pre-installed on all the nodes. Similarly, the nodes are also equipped with shadowing propagation model [24] which is used to simulate varying channel quality and multipath effects.

\subsection{Reconfiguration Cost}

The reconfiguration cost is presented in two aspects: the number of generated plans and the operation cost of each generated plan. The former is the representation of the cost in the generation phase and the latter reflects the cost of the network changing phase.

As stated in [12], the total reconfiguration cost incurred for each and every plan generated could be formulated by the following formula:

$$
C_{R_{i}}=p \alpha+q \beta+r \gamma
$$

In the formula, $p$ is the number of channel switch operations, $q$ is the number of radio switch and $r$ is the number of route detour operations involved in the reconfiguration plan $R_{i}$. Values of $\alpha, \beta$ and $\gamma$ denote the cost of the channel switch, radio switch and route switch respectively. If there are $n$ plans existing, $C_{R_{i}}$ is computed and stored for each and every plan.

Indicated by [12], the values of $\alpha, \beta$ and $\gamma$ are set to 5,7,10 respectively, and the costs of various combinations of primitive link changes involved in each plan are exhibited in Table 2

The columns CS, RdS and RtS denotes the number of the channel switch, radio switch and route switch respectively. $C_{R_{i}}$ indicates the total reconfiguration cost. Since the priorities of the same channel on different nodes are different, all the three operations are involved with priority change. In this case, the maximal priority involved in the reconfiguration plan is represented as MP in the table.

The objective of ALRS is to achieve maximum throughput with min-max priority of each link and we select the optimal plan by picking out plans with minimum MP 
Table 2: Configuration Cost for Feasible Plans

\begin{tabular}{|c|c|c|c|c|c|}
\hline No. & CS & RdS & RtS & $C_{R_{i}}$ & $\mathrm{MP}$ \\
\hline 1 & 1 & 0 & 0 & 5 & 4 \\
\hline 2 & 0 & 0 & 1 & 10 & 3 \\
\hline 3 & 1 & 0 & 1 & 15 & 2 \\
\hline 4 & 2 & 1 & 0 & 17 & 3 \\
\hline 5 & 0 & 1 & 2 & 27 & 2 \\
\hline 6 & 3 & 0 & 0 & 15 & 4 \\
\hline 7 & 0 & 0 & 3 & 30 & 3 \\
\hline
\end{tabular}

value. It is shown in Table 2 that plan 3 and 5 share the same MP and as we stated in Section V the plan with less cost is chosen as the optimal plan. In other words, plan 3 is the optimal plan generated by ALRS with the cost 15 .

In order to evaluate the cost of ALRS we make a comparative trial which is shown in Table 3

Table 3: Cost Comparison

\begin{tabular}{|c|c|c|}
\hline Method & Num of Feasible Plans & Optimal Cost $\left(C_{R_{i}}\right)$ \\
\hline ARS & 16 & 36 \\
\hline ERS & 16 & 15 \\
\hline ALRS & 7 & 15 \\
\hline
\end{tabular}

We tested the performance of ARS, ERS and ALRS respectively with the same simulating environment. One link is influenced and failed. The locations of nodes and gateways remain all the same and the reconfiguration range is set to 3 . We recorded the number of generated plans (namely feasible plans) and the optimal one for comparison. ARS and ERS both generated 16 feasible plans due to the exhaustive method while ALRS only generated 7 feasible plans. The cause of this phenomenon is that ALRS eliminates overlarge priority involved plans during the generation phase for saving time as we described in Section V. But the gateway involved reconfiguration plans are not influenced by this action and the throughput maximization is not impacted according to our proof. On the other hand, the min-max priority is not influenced apparently.

We calculated the costs of the optimal plans generated by all three methods as well 
and the result is shown in Table 3 too. ARS has not considered the cost of reconfiguration as the result indicates. ERS and ALRS share the same cost of the optimal plan because they selected the same optimal plan actually, and of course at a lower cost. There is possibility that the optimal plan generated by ERS is eliminated at the beginning of ALRS, and at that time a "sub-optimal" plan (in the view of cost) will be selected by ALRS. But the "sub-optimal" plan possesses higher maximal throughput or lower min-max priority which indicates less possibility of link failure in the future with no doubt due to the explanation of "loop effect". In this case, there is a trade-off among throughput, robustness and minimum cost and which one to choose is a matter of opinion and conjecture.

Due to the fact that the feasible plans generated by ALRS are not all-inclusive, it is unavoidable that there are some situations under which the optimal plan selected by ERS is eliminated by ALRS during the feasible plan generation phase. But except for the "loop effect", the behavior of the priority list achieves the same results as the cost analysis because well-performed and cost-effective channels are going to be given higher priorities. In this case, ALRS shares considerable possibility in generating the same optimal plan with ERS when "loop effect" is not occurred. On the other hand, faced with the "loop effect", ALRS outperforms ARS and ERS in efficiency, throughput and robustness. As a conclusion, ALRS possesses a large possibility in performing no less than ERS, let alone ARS.

\subsection{Evaluation Result}

This subsection presents the performance and analysis of ALRS. Available bandwidth and the probability of failure's occurrence are mainly considered in the evaluation. All the evaluation results are calculated as average values of 50-100 times of experiment.

\subsubsection{Throughput during recovery from one failure}

We first test our mechanism in traditional WMNs. In this case, the main cause of link failures is the unpredictable interference. As shown in Fig 6 ALRS outperforms ARS, ERS and greedy method both on throughput and recovery time. Due to the 


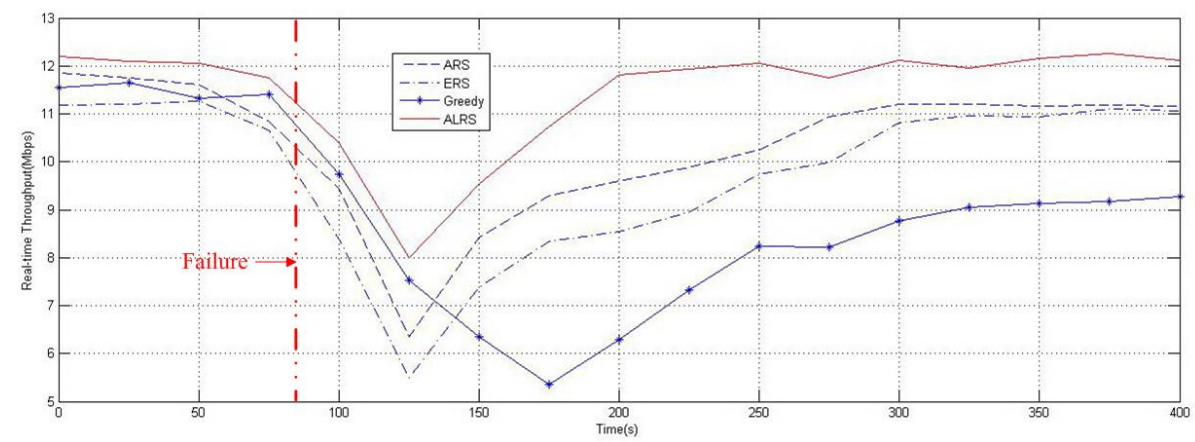

Figure 6: Real-time Throughput during Recovery from one failure

carefully designed mechanism, ALRS takes less time in generating feasible plans and achieves maximum throughput after reconfiguration.

When a failure happens, all of techniques we tested in the simulation experience rapid decrease on throughput performance. Note that ARS, ERS and ALRS almost rebound simultaneously while the Greedy method falls behind. However, ALRS actually experiences the fastest reaction time but not revealed in the figure due to the interval sampling. This is because ALRS generates relatively less reconfiguration plans than ERS and ARS. And that ALRS outperforms ARS and ERS on throughput reflects this fact in a way.

\subsubsection{Throughput during recovery from multiple failures}

It is a core promise of ALRS to deal with the continuous link failures, namely the "loop effect". In order to evaluate its performance on avoiding the "loop effect", we also arranged simulations on recovery from multiple continuous failures. As shown in Fig. 7, the performance of all four techniques when multiple link failure happened in a line is exhibited. Except for ALRS, the other three techniques all experience oscillating throughput. This is due to the fact that their designs are focusing on the recovery from one link failure. And unfortunately, they either generate an unsuitable plan for the subsequent failure recovery or get confused by the conflict of several reconfiguration plans. On the other hand, ALRS takes the priority into consideration, which actually reflects the subsequent failures that may be coming. As a result, ALRS recovers from 


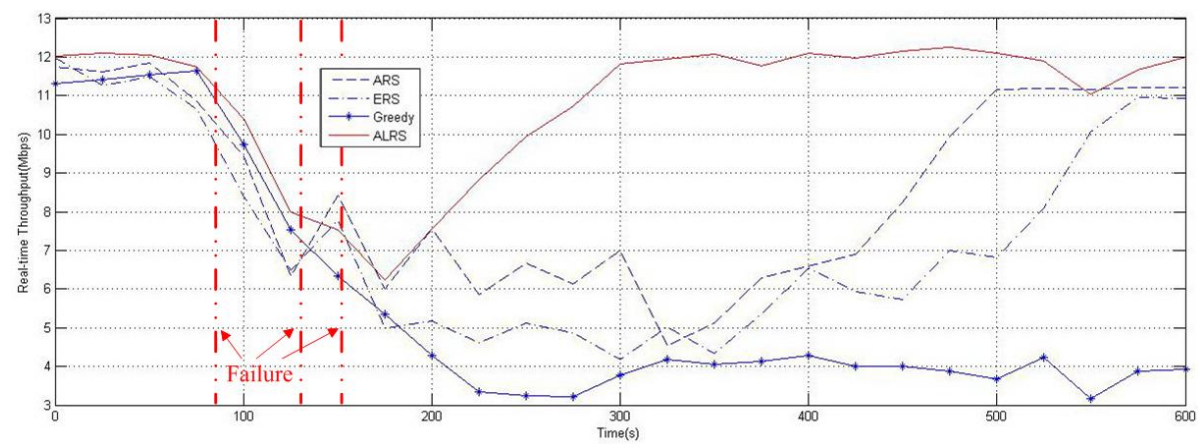

Figure 7: Real-time Throughput during Recovery from multiple failures

the multiple failures more quickly.

Notably, there are situations when ARS and ERS outperform ALRS (e.g. the time point when the third failure happens in Fig. 7f in our simulation. This phenomenon could be seen in single failure recovery simulations every now and then. Although the

number of reconfiguration plans generated in ALRS is no more than that in ARS and ERS, there are cases that they generate the same plans. At this time, ALRS should further consider the priority issues while ARS and ERS do not. Consequently, ARS and ERS outperform ALRS under such situation. However, Fig. 6 and 7 exhibit the average performance among plenty of simulations, and we could come to the point that ALRS outperforms the other techniques in general. But this is not an absolute conclusion in every situation.

\subsubsection{Throughput on the number of global channels}

The average throughput achieved after recovery from multiple failures on the number of global channels is also evaluated among the simulations, as shown in Fig 8 . Although ARS and ERS are not good at dealing with multiple failures during the recovery process, they may achieve good throughput performance after the reconfiguration, if there is only one faulty channel. However, they experience poor performance on throughput after recovery as the number of global channels increases. This is mostly due to the conflict of their chaotic reconfiguration plans on multiple channels. ALRS experiences the best performance not only during the recovery process, but also after 


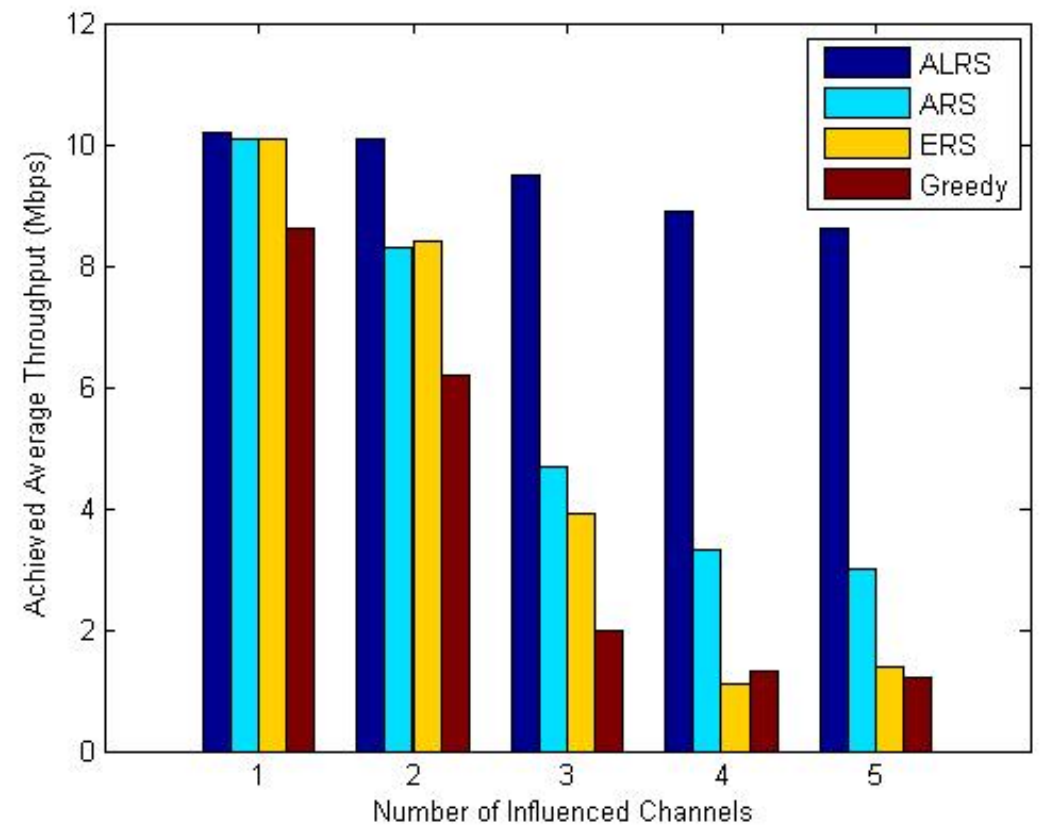

Figure 8: Average Throughput after Recovery from Multiple Failures on the Number of Global Channels

it.

\subsubsection{The performance on avoiding "loop effect"}

We also summarized the times of link failures on the number of global channels in the simulation. It is obvious that ALRS beats the other three techniques on "loop effect" avoidance. The result could be seen in Fig 9

According to Fig 8 and 9 , ALRS is able to make more flexible reconfiguration plans with more available channels. It considers the potential of future failures that may come and tries to avoid them during the reconfiguration plan generation. Since the plan with maximal throughput and min-max priority is chosen as the optimal plan, ALRS does not discriminate against the route switch operation. While on the other hand, the more channels there are, the less possible ARS and ERS choose to use a route switch, which makes the network suffer badly from the "loop effect". To sum up, ALRS efficiently avoids the "loop effect" and is able to achieve at least equivalent 


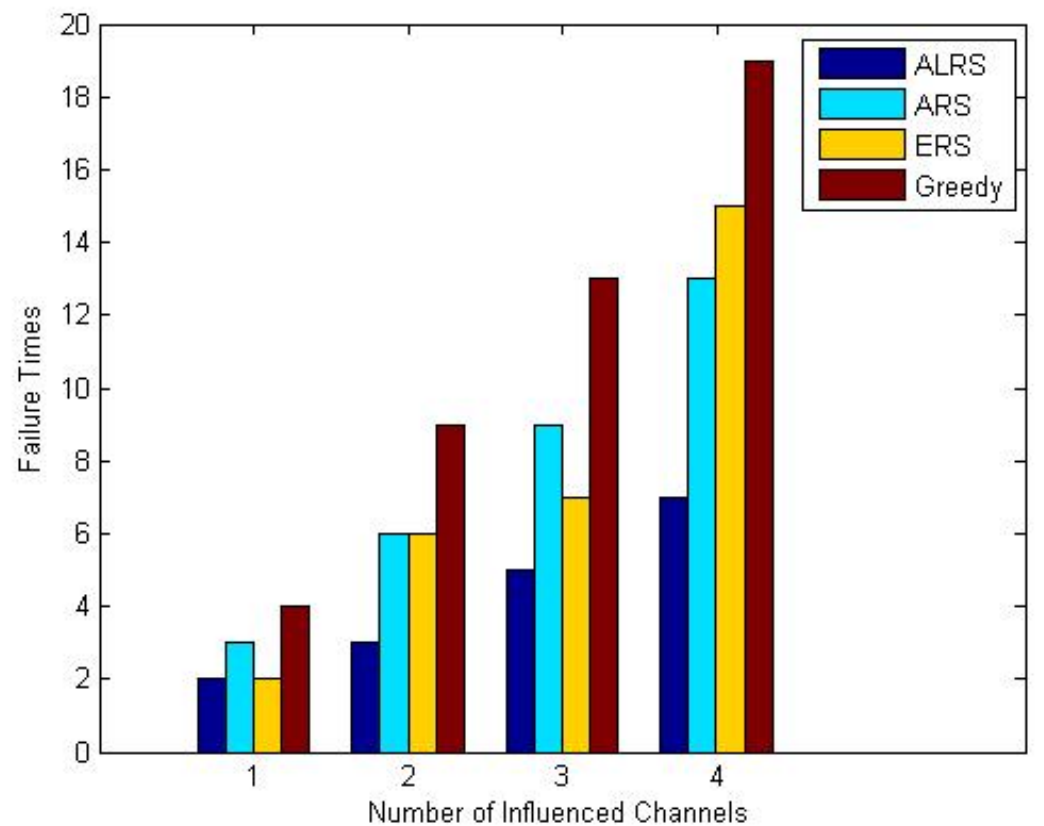

Figure 9: The Times of Link Failures on the Number of Global Channels

performance in general in the contrary with ARS and ERS.

\subsection{Data Analysis}

According to Fig 677/8, ALRS outperforms all the other competitors on the average throughput no matter during or after the reconfiguration, which means ALRS is more suitable for guaranteeing service continuity. Meanwhile, ALRS makes a significant contribution to practical use of municipal WMN due to the avoidance of the "loop effect", which is shown in Fig 7 /89 Thanks to the design of priority, ALRS is more adaptive in dealing with severe and volatile interference.

\subsection{ALRS's Advantages}

In this section, we compare and analyze the performances of ARS, ERS, ALRS and Greedy on throughput achievement and frequent link failures cause by "loop ef- 
average throughput during reconfiguration, recovery efficiency and robustness. ALRS experiences the least rebound time in all the simulations, and encounters the least link failures.

The relationship between the performance and the number of global channels is discussed in the simulation as well. ALRS achieves outstanding performance with several channels available and avoids the frequent link failures caused by "loop effect", which influences the performance of ARS and ERS, especially with more global channels.

The cost of ALRS and its comparison with ARS and ERS are considered and discussed, and ALRS takes the least time in generating feasible plans and possesses considerable possibility in achieving optimal cost in reconfiguration.

Although the objective of ALRS is to achieve maximum throughput and the avoidance of "loop effect", ALRS performs perfectly in dealing with normal occasional link failure as well.

\section{CONCLUSION}

In this section, we will give a brief summary of our work and experiment. Then we will discuss some future work.

\subsection{Work Summarize}

We propose a scheme for municipal link recovery called "Anti-Loop Reconfiguration System" in this paper. Two techniques namely ARS and ERS have already achieved self-adaptive reconfiguration, but the ignorance of "loop effect" makes them unsuitable for municipal use. ALRS not only overcomes the "loop effect", but also takes throughput maximum into consideration, both of which make ALRS outperform all the existing techniques and the best choice for municipal WMNs. Moreover, ALRS is not limited by the number of antennas in the mesh node, thanks to which we are able to deploy ALRS to any existing wireless mesh network breezily.

In our experiment, we achieved dramatic experimental results on calculating average performance. But we have to declare that our scheme cannot exceed other techniques in each single simulation. Moreover, the greedy method which can be viewed 
as the worst technique in the experiment may experience the best performance under particular simulations, due to the uncertainty of the network and interference around the laboratory.

We are convinced that the average performance is the loyal reflection of techniques because of our carefully designed simulation.

\subsection{Future Work}

Besides throughput, more QoS constraints will be taken into account for link recovery research such as delay, packet loss rate, etc. Meanwhile, other reasons except for block and interference which may cause "loop effect" are also attracting us for further study.

In wireless mesh networks, there are more and more studies working on flow level as the trend of delicate management rises, inspired by which, we are curious in reformulating the link recovery problem on flow level with continuity of transmission guaranteed. We will carry on looking for the advantages of flow management and its reflection in link recovery.

\section{Acknowledgement}

This paper is supported by the National Science Foundation of China under Grant No. U1301256, 61170058, 61272133, 61472383 and 51274202, Special Project on IoT of China NDRC (2012-2766), China Postdoctoral Science Foundation project under Grant No. 2012T50547, Research Fund for the Doctoral Program of Higher Education of China No. 20123402110019, the NSF of Jiangsu Province Grant No. BK2012632, and Suzhou Industry Fund Grant No. SYG201302.

\section{References}

[1] J. Tang, G. Xue, W. Zhang, Maximum throughput and fair bandwidth allocation in multi-channel wireless mesh networks., in: INFOCOM, 2006, pp. 1-10. 
[2] B. Leng, L. Huang, H. Xu, C. Yang, X. Wang, A self-adaptive reconfiguration scheme for throughput maximization in municipal wmns, in: Quality of Service (IWQoS), 2015 IEEE 23rd International Symposium of, IEEE, 2015.

[3] I. F. Akyildiz, X. Wang, A survey on wireless mesh networks, Communications Magazine, IEEE 43 (9) (2005) S23-S30.

[4] K. Pendke, A. Dhamgaye, The survey of techniques for link recovery \& admission control in wireless mesh network, International Journal of Computer Science and Mobile Computing 3 (1) (2014) 150-152.

[5] R. Bhatia, L. Li, Throughput optimization of wireless mesh networks with mimo links, in: INFOCOM 2007. 26th IEEE International Conference on Computer Communications. IEEE, IEEE, 2007, pp. 2326-2330.

[6] P. Kyasanur, N. H. Vaidya, Capacity of multi-channel wireless networks: impact of number of channels and interfaces, in: Proceedings of the 11th annual international conference on Mobile computing and networking, ACM, 2005, pp. 43-57.

[7] K. N. Ramachandran, E. M. Belding-Royer, K. C. Almeroth, M. M. Buddhikot, Interference-aware channel assignment in multi-radio wireless mesh networks., in: INFOCOM, Vol. 6, 2006, pp. 1-12.

[8] W. Tu, A multi-rate multi-channel multicast algorithm in wireless mesh networks, in: Local Computer Networks (LCN), 2014 IEEE 39th Conference on, IEEE, 2014, pp. 55-63.

[9] C.-c. Chen, C. Chekuri, Urban wireless mesh network planning: The case of directional antennas, Tech. rep., Illinois University (2007).

[10] M. Alicherry, R. Bhatia, L. E. Li, Joint channel assignment and routing for throughput optimization in multi-radio wireless mesh networks, in: Proceedings of the 11th annual international conference on Mobile computing and networking, ACM, 2005, pp. 58-72. 
[18] K. N. Ramachandran, E. M. Belding-Royer, K. C. Almeroth, M. M. Buddhikot, Interference-aware channel assignment in multi-radio wireless mesh networks., in: INFOCOM, Vol. 6, 2006, pp. 1-12.

[19] A. Raniwala, T.-c. Chiueh, Architecture and algorithms for an ieee 802.11-based

[11] K.-H. Kim, K. G. Shin, Self-reconfigurable wireless mesh networks, Networking, IEEE/ACM Transactions on 19 (2) (2011) 393-404.

[12] R. Ramakrishnan, N. Sankar Ram, O. A. Alheyasat, A cost aware reconfiguration technique for recovery in wireless mesh networks, in: Recent Trends In Information Technology (ICRTIT), 2012 International Conference on, IEEE, 2012, pp.

[13] I. F. Akyildiz, X. Wang, W. Wang, Wireless mesh networks: a survey, Computer networks 47 (4) (2005) 445-487.

[14] G. Murugaboopathi, T. Rathishbabu, R. Venkatesh, An analysis of reconfiguration approaches for recovery in wireless mesh networks, International Journal of

[15] D. B. Johnson, D. A. Maltz, Dynamic source routing in ad hoc wireless networks, in: Mobile computing, Springer, 1996, pp. 153-181.

[16] G. Lu, B. Krishnamachari, C. S. Raghavendra, An adaptive energy-efficient and low-latency mac for data gathering in wireless sensor networks, in: Parallel and Distributed Processing Symposium, 2004. Proceedings. 18th International, IEEE, 2004, p. 224.

[17] A. Brzezinski, G. Zussman, E. Modiano, Enabling distributed throughput maximization in wireless mesh networks: a partitioning approach, in: Proceedings of the 12th annual international conference on Mobile computing and networking, ACM, 2006, pp. 26-37. multi-channel wireless mesh network, in: INFOCOM 2005. 24th Annual Joint Conference of the IEEE Computer and Communications Societies. Proceedings IEEE, Vol. 3, IEEE, 2005, pp. 2223-2234. 
[20] M. Doraghinejad, H. Nezamabadi-Pour, A. Mahani, Channel assignment in multiradio wireless mesh networks using an improved gravitational search algorithm, Journal of Network and Computer Applications 38 (2014) 163-171.

[21] A. Tanenbaum, M. Steen, Distributed systems principles and designs (2007).

[22] "the network simulator-ns-2", http://www.isi.edu/nsnam/ns (. Retrieved 2015-02-06.).

[23] R. Draves, J. Padhye, B. Zill, Routing in multi-radio, multi-hop wireless mesh networks, in: Proceedings of the 10th annual international conference on Mobile computing and networking, ACM, 2004, pp. 114-128.

[24] T. S. Rappaport, et al., Wireless communications: principles and practice, Vol. 2, prentice hall PTR New Jersey, 1996. 